Internat. J. Math. \& Math. Sci.

Vol. 24, No. 8 (2000) 539-548

S0161171200002854

(c) Hindawi Publishing Corp.

\title{
ON THE MISLIN GENUS OF CERTAIN CIRCLE BUNDLES AND NONCANCELLATION
}

\author{
PETER HILTON and DIRK SCEVENELS
}

(Received 1 February 1999)

\begin{abstract}
In an earlier paper, the authors proved that a process described much earlier for passing from a finitely generated nilpotent group $N$ of a certain kind to a nilpotent space $X$ of finite type produced a bijection of Mislin genera $\mathscr{G}(N) \cong \mathscr{G}(X)$. The present paper is concerned with related results obtained by weakening the restrictions on $N$ and generalizing the homotopical nature of the spaces $X$ to be associated with a given $N$.
\end{abstract}

Keywords and phrases. Mislin genus, circle bundles, noncancellation, nilpotent groups, nilpotent spaces.

2000 Mathematics Subject Classification. Primary 55P60, 20 F18.

1. Introduction. The genus of a finitely generated nilpotent group $N$ was introduced in [11] as the set of isomorphism classes of finitely generated nilpotent groups $M$ such that the localizations $M_{p}$ and $N_{p}$ are isomorphic at every prime $p$. Analogously the Mislin genus of a connected nilpotent space $X$ (of the homotopy type of a CW-complex) of finite type (cf. [4]) is defined as the set of homotopy types of nilpotent spaces $Y$ of finite type such that the localizations $Y_{p}$ and $X_{p}$ are homotopy equivalent at every prime $p$.

In [10] the author, generalizing results in [13], provided a powerful tool for the calculation of the Mislin genus $\mathscr{G}(X)$ of a (connected) nilpotent space $X$ of finite type which was an $H_{0}$-space (i.e., its rationalization is an $H$-space) and had only finitely many nonvanishing homotopy groups. It turns out that $\mathscr{G}(X)$ may then be given the structure of a finite abelian group. Much earlier, in [1, 2], the Mislin genus $\mathscr{G}(N)$ of a finitely generated nilpotent group $N$ satisfying three conditions had been calculated. The conditions are stated in terms of the natural short exact sequence

$$
T N \longrightarrow N \rightarrow F N
$$

where $T N$ is the torsion subgroup of $N$ with $F N$ the torsionfree quotient; they are

(i) $T N$ and $F N$ are commutative;

(ii) relation (1.1) splits for the action $\omega: F N \rightarrow$ Aut $T N$;

(iii) $\omega(F N)$ lies in the center of Aut $T N$.

It was observed in [1] that, in the presence of (i), (iii) is equivalent to

(iii') for all $\xi$ in $F N$, there exists an integer $u$ such that $\xi \cdot a=\omega(\xi)(a)=u a$ for all $a \in T N$. (Here, $T N$ is written additively.)

A finitely generated nilpotent group satisfying (i), (ii), (iii) is also said to belong to $\mathcal{N}_{1}$. 
Now it was known (see $[3,11])$ that if $F N$ is commutative, then $\mathscr{G}(N)$ may also be given the structure of a finite abelian group. Moreover, a procedure was described in [2], and exploited in [6], whereby one might associate with the group $N$, in the case that $F N$ is cyclic, a circle bundle $X$, over a base $M$ which depends only on $\mathscr{G}(N)$, inducing an injection $\mathscr{G}(N) \longrightarrow \mathscr{G}(X)$. Using [10, McGibbon's formula], it was shown in [6] that the injection is indeed a bijection; in fact, the abelian group structures on $\mathscr{G}(N)$ and $\mathscr{G}(X)$ coincide. However, it should be remarked that the result in [6] was based on the assumption that one had chosen $X$ to have vanishing homotopy groups in dimensions $i \geq 3$.

The restriction on $N$, that $F N$ be cyclic, is reasonable if one wishes to construct and calculate examples of nontrivial genera, since a result of [8] implies that $\mathscr{G}(N)$ is trivial if $F N$ is not cyclic. On the other hand, from the point of view of obtaining information about the genus of a certain space $X$, it is significant that we can extend the association $N \mapsto X$ to the case of $F N$ not cyclic and hence, via McGibbon's formula, show that $\mathscr{G}(X)=0$ in this case. We do this in Section 2. However, by refining the result quoted from [8], we obtain very precise information about the nature of the spaces $Y$ of which we can claim the triviality of the genus. The improvement of the result from [8] is described in Section 6, and the spaces in question are specified in Theorems 2.3 and 2.4 .

In Section 3, we describe an enlargement of the domains of validity of our main result in [6] and our results in Section 2. The injection $\mathscr{G}(N)>>\mathscr{G}(X)$ referred to earlier only requires that the base $M$ of the circle bundle $X$ have specified 2-type (i.e., we specify $\pi_{1} M, \pi_{2} M$ and the action of $\pi_{1} M$ on $\pi_{2} M$ as functions of $N$ ), and says nothing about the homotopy groups of $M$ in dimensions $i \geq 3$ beyond requiring that $M$ (and hence $X$ ) be nilpotent. On the other hand, we have no reason to expect the injection to be a bijection in this generality. We describe in this section how we may ease the restriction on $X$ (that its higher homotopy groups vanish) and still preserve the bijective property proved in [6] (when $F N$ is cyclic) and in Section 2 (when $F N$ is not cyclic).

In Section 4, we extend the method of [6] and this paper from $\mathscr{G}(N)$, where $N$ is a finitely generated nilpotent group in $\mathcal{N}_{1}$, to $\mathscr{G}\left(N^{k}\right)$, where $N^{k}$ is the direct product of $k$ copies of $N$. This extension is significant because $N^{k}$, with $k \geq 2$, does not inherit property (iii) from $N$, though $\mathscr{G}\left(N^{k}\right)$ is still a finite abelian group since $N^{k}$ inherits property (i) from $N$. The calculation of $\varphi\left(N^{k}\right)$ for $k \geq 2$, was carried out in [7]-it was already known that there is a surjection $\mathscr{G}(N) \rightarrow \mathscr{G}\left(N^{k}\right)$ given by $\tilde{N} \mapsto \tilde{N} \times N^{k-1}$ (cf. [1])and effectively we show in Section 4 that exactly the same process of passing to the appropriate quotient groups takes place in analysing the surjection $\mathscr{G}(X) \rightarrow \mathscr{G}\left(X^{k}\right)$, where $X^{k}$ is the topological product of $k$ copies of $X$, the circle bundle associated with $N$. As a consequence, we know that $\mathscr{G}\left(N^{k}\right) \rightarrow \mathscr{G}\left(X^{k}\right)$ is bijective. Of course, we can here allow the generalization of the construction of $X$ from $N$ discussed in Section 3 . We conjecture that we may further generalize the results of this section by replacing $N^{k}$ by $N_{1} \times N_{2} \times \cdots \times N_{k}$, where each $N_{i}$ is a finitely generated nilpotent group satisfying conditions (i), (ii), and (iii); more precisely, we conjecture that we obtain a bijection $\mathscr{G}\left(N_{1} \times N_{2} \times \cdots \times N_{k}\right) \cong \mathscr{G}\left(X_{1} \times X_{2} \times \cdots \times X_{k}\right)$. The abelian group $\mathscr{G}\left(N_{1} \times N_{2} \times \cdots \times N_{k}\right)$ was calculated in [5]. 
In Section 5, we use the equivalence between $\mathscr{G}\left(N^{k}\right)$ and $\mathscr{G}\left(X^{k}\right)$ to transfer noncancellation phenomena from the category of finitely generated nilpotent groups to that of nilpotent spaces of finite type. The original noncancellation phenomena were described in [9]; they are a ready consequence of the calculation of $\mathscr{G}\left(N^{k}\right)$.

2. The case $F N$ noncyclic. Here we generalize the procedure used in [6] to study the case when $F N$ is not cyclic. Thus we have, as in Section 1, a finitely generated nilpotent group $N$ belonging to $\mathcal{N}_{1}$ and fitting into a split short exact sequence

$$
T N>N \longrightarrow F N,
$$

but now we assume that $F N$ is free abelian of rank $r \geq 2$. We then know that we can write

$$
F N=\left\langle\xi_{1}, \xi_{2}, \ldots, \xi_{r}\right\rangle
$$

where, for $i=1,2, \ldots, r$,

$$
\xi_{i} \cdot a=u_{i} a, \quad \forall a \in T N,
$$

and the order of $u_{i} \bmod m$ is $t_{i}$, where $m=\exp T N$, and

$$
t_{1}\left|t_{2}\right| \cdots \mid t_{r}
$$

As in [1], we know that the genus of $N$ is given by

$$
\mathscr{G}(N) \cong\left(\mathbb{Z} / t_{1}\right)^{*} /\{ \pm 1\} .
$$

However, [8, Theorem 1.1] tells us that, in fact, $t_{1}=1$ or 2 (given $r \geq 2$ ), so that we have the following.

THEOREM 2.1. If $r \geq 2$, then $\mathscr{G}(N)$ is trivial.

Actually, as will be shown in Section 6, we can improve on [8, Theorem 1.1] and deduce (see Theorem 6.1) the following.

THEOREM 2.2. If $r \geq 2$, the quantities $t_{1}, t_{2}, \ldots, t_{r}$ of (2.4) satisfy

$$
t_{1}=t_{2}=\cdots=t_{r-2}=1, \quad t_{r-1}=1 \text { or } 2 .
$$

In fact, the case $t_{r-1}=2$ is a highly exceptional case-though it certainly does occur. Let us consider the group $N_{0}$ in $\mathcal{N}_{1}$, where $\exp T N_{0}=m$ and $F N_{0}$ is cyclic with generator $\xi$ and $\xi \cdot a=u a$ for all $a \in T N_{0}$. Suppose that the order of $u \bmod m$ is $t$, so that

$$
\mathscr{G}\left(N_{0}\right) \cong(\mathbb{Z} / t)^{*} /\{ \pm 1\} .
$$

Then, with $t_{r}=t$, the group $N$ we have been discussing, with $t_{1}=t_{2}=\cdots=t_{r-1}=1$, $t_{r}=t$, is just $C^{r-1} \times N_{0}$, where $C$ is cyclic infinite, so that

$$
\mathscr{G}\left(C^{r-1} \times N_{0}\right)=0 \text { for } r \geq 2 .
$$


Now suppose that $N_{0}$ gives rise, as in [6], to the circle bundle $X_{0}$ over a base $M$, where $\pi_{1} X_{0}=C, \pi_{2} X_{0}=T N_{0}$, and $\pi_{i} X_{0}=0$ for $i \geq 3$. Then, as proved in [6],

$$
\mathscr{G}\left(X_{0}\right) \cong(\mathbb{Z} / t)^{*} /\{ \pm 1\} .
$$

We can now carry out a process very like that in [6]. Using $X_{0}$ as our base, we construct a trivial circle bundle over $X_{0}$ and the argument of [6] show that

$$
\mathscr{G}\left(C \times N_{0}\right) \cong \mathscr{G}\left(S^{1} \times X_{0}\right) .
$$

Iterating this procedure, we finally obtain the following.

THEOREM 2.3. We have $\mathscr{G}\left(\left(S^{1}\right)^{r-1} \times X_{0}\right)=0$ for $r \geq 2$.

Let us now turn to the exceptional case where $t_{r-1}=2$ in Theorem 2.2. We first recall when the exceptional case arises. Using the notation above (and in Section 6), let

$$
m=p_{1}^{n_{1}} p_{2}^{n_{2}} \cdots p_{\lambda}^{n_{\lambda}}
$$

where $p_{1}<p_{2}<\cdots<p_{\lambda}$ are primes. Moreover, we know that each $u_{i}$ is of the form

$$
u_{i}=1+c_{i} p_{1}^{n_{i 1}} p_{2}^{n_{i 2}} \cdots p_{\lambda}^{n_{i \lambda}} \text {, with } n_{i j} \geq 1 \text { for } j=1,2, \ldots, \lambda,
$$

where $c_{i}$ is prime to $p_{1} p_{2} \cdots p_{\lambda}$. Then we may find ourselves in the exceptional case of Theorem 6.1, with $\varepsilon=2$, if $p_{1}=2$ and $n_{1} \geq 3$.

Let us then begin with a group $N$ in $\mathcal{N}_{1}$ such that $r=2$ and $t_{1}=2, t_{2}=t$. We construct a nilpotent $C W$-complex $M$ according to the following homotopical specifications:

- $\pi_{1} M=C_{2} \times C=\langle\eta, \xi\rangle$;

- $\pi_{2} M=T N$, with $\eta \cdot a=2 a$, and $\xi \cdot a=t a$, for all $a \in T N$;

- $\pi_{i} M=0$ for $i \geq 3$.

Then $H^{2}(M ; \mathbb{Z}) \supseteq \operatorname{Ext}\left(H_{1} M, \mathbb{Z}\right)=\operatorname{Ext}(\mathbb{Z} / 2, \mathbb{Z})=\mathbb{Z} / 2=\langle g\rangle$, and we interpret $g$ as the homotopy class of a map, which, by abuse, we also designate as $g: M \rightarrow K(\mathbb{Z}, 2)$. We then use $g$ to induce a circle bundle $X$ over $M$; thus,

$$
S^{1} \rightarrow X \stackrel{h}{\rightarrow} M \stackrel{g}{\rightarrow} K(\mathbb{Z}, 2) .
$$

Then $h$ induces an isomorphism $h_{*}: \pi_{i} X \cong \pi_{i} M$ for $i \geq 2$; moreover, $\pi_{1} X=C \times C$ and $h$ maps one generator of $\pi_{1} X$ onto $\eta$, the other being mapped onto $\xi$. As in [6] (cf. [12, Theorem 1.8]), we have

$$
N \cong\left[S^{1}, \Omega X\right]_{\mathrm{fr}}=\pi_{2} X \rtimes \pi_{1} X,
$$

the semidirect product for the action of $\pi_{1} X$ on $\pi_{2} X$. It again follows, just as we argued in [6], that $\mathscr{G}(N) \cong \mathscr{G}(X)$. However, $\mathscr{G}(N)=0$, so we conclude that

$$
\mathscr{G}(X)=0 .
$$

However, we may now put $X$ in the role of $M$ and repeat the construction using the zero element of $H^{2}(X ; \mathbb{Z})$. We obtain, of course, the trivial bundle $S^{1} \times X$, corresponding to the nilpotent group $C \times N$, where, of course, $\mathscr{G}(C \times N)=0$. Continuing in this way, we finally prove the following. 
THEOREM 2.4. Let $N$ be as above, and let $X$ be the associated circle bundle constructed as in (2.13). Then, for any $r \in \mathbb{N}$,

$$
\mathscr{G}\left(\left(S^{1}\right)^{r} \times X\right)=0 .
$$

3. Carrying the calculation of $\mathscr{G}(X)$ further. Let us remove a key item in the specification of $M$ in [6] and in Section 2. Thus we first assume that $F N$ in (2.1) is cyclic, but we no longer insist that $\pi_{i} M=0$ for $i \geq 3$. In fact, we impose no restriction at all on the homotopy groups of $M$ in dimensions $i \geq 3$ as $\pi_{1} M$-modules, beyond insisting that $M$ be nilpotent. We may then construct $X$ exactly as we did in [6], and it remains true that we may find, for each $N_{\ell}$ in the genus of $N$, a space $X_{\ell}$ in the genus of $X$, and that we obtain thereby an embedding of $\mathscr{G}(N)$ in $\mathscr{G}(X)$. (Indeed, the method of passing from $N$ to $X$ was carried out in [2] in the sense described in this paragraph, that is, without requiring that $\pi_{i} M=0$ for $1 \geq 3$.)

However, at this level of generality we certainly cannot claim that $\mathscr{G}(N)$ and $\mathscr{G}(X)$ coincide, i.e., the embedding referred to above is a bijection.

Since we certainly want to apply the key formula of McGibbon, we must insist that $X$ be an $H_{0}$-space with only finitely many nonzero homotopy groups. We will be content to present here a generalization of the theorem of [6] which requires virtually no change in the arguments. We use the notation of Section 1; and denote by $T$ the set of primes $p$ such that $N$ has $p$-torsion.

THEOREM 3.1. Let $N \in \mathcal{N}_{1}$ with $F N$ cyclic and let $M$ be constructed according to the following specifications:

- $\pi_{1} M=C_{t}=\langle\eta\rangle$;

- $\pi_{2} M=T N$, with $\eta \cdot a=u a$, for all $a \in T N$;

- $\pi_{i} M=0$, for almost all $i$ and is always a (finite) $T$-group if $i \geq 2$.

One then constructs $X$ as in [6] and the mapping $N_{\ell} \mapsto X_{\ell}$, for $\ell \in(\mathbb{Z} / t)^{*}$, induces a bijection of $\mathscr{G}(N)$ with $\mathscr{G}(X)$.

Proof. We only need to remark that $X$ is an $H_{0}$-space, indeed, $X$ is rationally equivalent to $S^{1}$, and that $X$ has only finitely many nonzero homotopy groups since $\pi_{i} X \cong \pi_{i} M$ for $i \geq 2$. Thus we may apply McGibbon's sequence

$$
s \text {-Equ } X \stackrel{d}{\rightarrow}(\mathbb{Z} / s)^{*} /\{ \pm 1\} \rightarrow \varphi(X)
$$

to calculate $\mathscr{G}(X)$, except that $s$ may be replaced by a larger $T$-number $\tilde{s}$ having $S$ as a factor. However, both an $s$-equivalence and an $\tilde{s}$-equivalence of $X$ are just a $T$ equivalence; and the calculation of $\mathscr{G}(X)$ from (3.1) is unaffected by replacing $s$ in the middle term of (3.1) by any other $T$-number having $s$ as a factor. Thus we still obtain, from the calculations in [6], the conclusion $\mathscr{G}(X) \cong(\mathbb{Z} / t)^{*} /\{ \pm 1\}$, whence, finally, $\mathscr{G}(N) \cong \mathscr{G}(X) \cong(\mathbb{Z} / t)^{*} /\{ \pm 1\}$.

4. Products. In this section, we will calculate the Mislin genus of the product $X^{k}$ of $k$ copies of $X$ for $k \geq 2$, where $X$ is a circle bundle associated with a nilpotent group $N$ in $\mathcal{N}_{1}$ by the methods of [6] or Section 2. 
We start by a very general result.

LEMMA 4.1. Let $k \geq 1$. If $X$ is a connected, nilpotent, finite type $H_{0}$-space with at most a finite number of nonzero homotopy groups, then there is an epimorphism

$$
\rho: \mathscr{G}(X) \longrightarrow \mathscr{G}\left(X^{k}\right) .
$$

Proof. By [10, McGibbon's result], and using the notation of the paper (except that we replace McGibbon's $t$ by $s$, since we have used $t$ for another purpose), we know that there are exact sequences

$$
\begin{gathered}
s \text {-Equ } X \stackrel{d}{\rightarrow}\left[(\mathbb{Z} / s)^{*} /\{ \pm 1\}\right]^{\ell} \longrightarrow \mathscr{G}(X), \\
\tilde{s} \text {-Equ } X^{k} \stackrel{\tilde{d}}{\longrightarrow}\left[(\mathbb{Z} / \tilde{s})^{*} /\{ \pm 1\}\right]^{\ell} \rightarrow \mathscr{G}\left(X^{k}\right) .
\end{gathered}
$$

By definition it is easily seen that $\tilde{s}=s$, so that there is a diagram

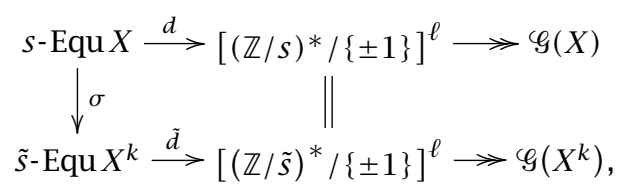

where $\sigma$ is given by $\sigma(\phi)=\phi \times$ id. Of course, $\tilde{d} \circ \sigma=d$ in (4.3), so that an epimorphism $\rho: \mathscr{G}(X) \rightarrow \mathscr{G}\left(X^{k}\right)$ is induced in (4.3).

In particular, we infer from Theorems 2.3 and 2.4 the following result.

Proposition 4.2. Let $Y$ be any of the spaces discussed in Theorems 2.3 and 2.4. Then $\varphi\left(Y^{k}\right)=0$ for $k \geq 1$.

In the case of a group $N$ in $\mathcal{N}_{1}$ with $F N$ cyclic, the genus of the direct product $N^{k}$ was calculated in [7]. More precisely, suppose, according to [1], that

$$
\mathscr{G}(N) \cong(\mathbb{Z} / t)^{*} /\{ \pm 1\},
$$

where

$$
t=p_{1}^{\ell_{1}} \cdots p_{\lambda}^{\ell_{\lambda}}
$$

with $p_{1}<p_{2}<\cdots<p_{\lambda}$ prime numbers, and $\ell_{i} \geq 1$ for $i \in\{1, \ldots, \lambda\}$. Starting with the exact sequence

$$
T \text {-Aut } N^{k} \longrightarrow(\mathbb{Z} / e)^{*} /\{ \pm 1\} \rightarrow \mathscr{G}\left(N^{k}\right),
$$

where $T$ is the set of primes occuring in the torsion of $N$, so that $t$ is a $T$-number, it is shown in [7] that

$$
\mathscr{G}\left(N^{k}\right) \cong \frac{\mathscr{G}(N)}{H}
$$


where, after the identification (4.4), $H$ consists of those residue classes $m \bmod t$ such that

$$
m \equiv \pm 1 \bmod p_{i}^{\ell_{i}} \quad \forall i \in\{1, \ldots, \lambda\} .
$$

Repeating the construction of the circle bundle $X$ associated with $N$ (cf. [6]), and considering products of these, we obtain the torus fibration

$$
\left(S^{1}\right)^{k} \longrightarrow X^{k} \longrightarrow M^{k} \stackrel{g \times \cdots \times g}{\longrightarrow} K(\mathbb{Z}, 2) \times \cdots \times K(\mathbb{Z}, 2) .
$$

Again, as in [6], we have that $N^{k} \cong\left[S^{1}, \Omega\left(X^{k}\right)\right]_{\mathrm{fr}}$, the group of free homotopy classes; and the short exact sequences

$$
T N^{k}>N^{k} \longrightarrow F N^{k}, \quad \pi_{2} X^{k}>\left[S^{1}, \Omega\left(X^{k}\right)\right]_{\mathrm{fr}} \rightarrow \pi_{1} X^{k}
$$

may be identified. To complete the calculation of $\varphi\left(X^{k}\right)$, we have to compare the exact sequence (cf. [10])

$$
s \text {-Equ } X^{k} \stackrel{d}{\longrightarrow}(\mathbb{Z} / s)^{*} /\{ \pm 1\} \rightarrow \mathscr{G}\left(X^{k}\right)
$$

with (4.6). Since $s=\exp T N$ (cf. [6]), we infer that $s$-Equ $X^{k}=T$-Equ $X^{k}$. It remains to analyze $d$ in (4.11). Again, as in [6], it is clear that any $f \in s$-Equ $X^{k}$ induces a commutative diagram

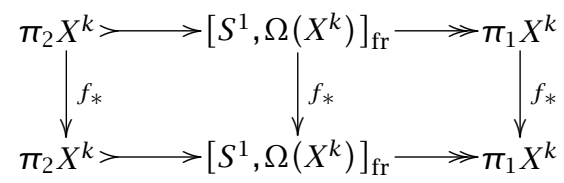

or, equivalently,

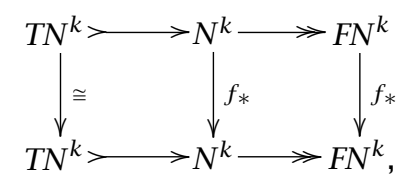

where $f_{*}: F N^{k} \rightarrow F N^{k}$ is a $T$-automorphism. Thus $\operatorname{det} f_{*} \equiv m \bmod t$, where $m \in H$, as defined in (4.8), by [7, Proposition 2.2]. Conversely, given any residue class $m$ mod $t$ satisfying (4.8), we can construct a $T$-equivalence $f: X^{k} \rightarrow X^{k}$ such that $d(f)=$ $\operatorname{det}\left(f_{*}: \pi_{1} X^{k} \rightarrow \pi_{1} X^{k}\right)=m$. Indeed, we use the construction of the homomorphism $\psi: F N^{k} \rightarrow F N^{k}$ in [7, proof of Theorem 1.3] to obtain a map

$$
\psi: K(\mathbb{Z}, 2) \times \cdots \times K(\mathbb{Z}, 2) \longrightarrow K(\mathbb{Z}, 2) \times \cdots \times K(\mathbb{Z}, 2),
$$

making the diagram

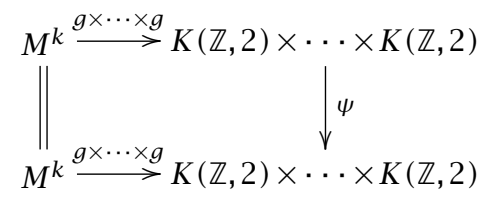


commutative. This yields a commutative diagram

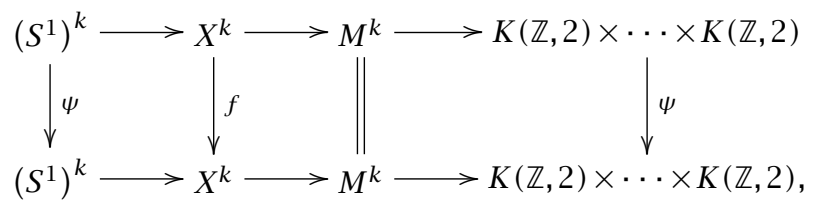

and $f$ is the desired $T$-equivalence. Concluding, we thus have proved the following theorem.

THEOREM 4.3. Let $N \in \mathcal{N}_{1}$ with $F N$ cyclic, and let $X$ be the associated circle bundle, as constructed in [6]. Then $\mathscr{G}\left(X^{k}\right)$ for $k \geq 2$ is obtained from $\varphi(X) \cong(\mathbb{Z} / t)^{*} /\{ \pm 1\}$ by factoring out those residue classes $m \bmod t$ satisfying (4.8). Indeed, $\mathscr{G}\left(X^{k}\right) \cong \mathscr{G}\left(N^{k}\right)$.

Observe that, instead of constructing the circle bundle $X$ and using the arguments of [6], we could have used the arguments of Section 3, thus allowing $X$ to have some nonzero higher homotopy groups, and that the results of Proposition 4.2 and Theorem 4.3 would remain true.

5. Noncancellation phenomena. Let $N \in \mathcal{N}_{1}$ with $F N$ cyclic, and let $X$ be the associated circle bundle. According to [1, 6],

$$
\mathscr{G}(N) \cong \mathscr{G}(X) \cong \frac{(\mathbb{Z} / t)^{*}}{\{ \pm 1\}},
$$

where $t$ is as in (4.5). Furthermore, a complete set $\left\{X_{\ell} \mid \ell \in(\mathbb{Z} / t)^{*}\right\}$ of homotopy types of spaces in $\mathscr{G}(X)$, in bijective correspondence with the complete set $\left\{N_{\ell} \mid \ell \in(\mathbb{Z} / t)^{*}\right\}$ of isomorphism classes in $\mathscr{G}(N)$, was described in [6]. Using the results of the previous section, we derive some consequences regarding noncancellation phenomena, paralleling results obtained in [9].

THEOREM 5.1. Let $k \geq 2$ and let $m_{1}, \ldots, m_{k}, m_{1}^{\prime}, \ldots, m_{k}^{\prime} \in(\mathbb{Z} / t)^{*}$. The following conditions are equivalent:

- $X_{m_{1}} \times \cdots \times X_{m_{k}} \simeq X_{m_{1}^{\prime}} \times \cdots \times X_{m_{k}^{\prime}}$;

- $N_{m_{1}} \times \cdots \times N_{m_{k}} \cong N_{m_{1}^{\prime}} \times \cdots \times N_{m_{k}^{\prime}}$;

- there exists a residue class $m$ mod $t$ satisfying (4.8) such that

$$
m_{1} \cdots m_{k} \equiv m m_{1}^{\prime} \cdots m_{k}^{\prime} \bmod t
$$

Proof. This is essentially a consequence of our proof of Theorem 4.3.

COROLlarY 5.2. Let $k \geq 1$ and let $m_{1}, m_{2} \in(\mathbb{Z} / t)^{*}$. Then $X_{m_{1}} \times X^{k} \simeq X_{m_{2}} \times X^{k}$ if and only if there exists a residue class $m \bmod t$ satisfying (4.8) such that $m_{1} \equiv$ $m m_{2} \bmod t$.

As a final consequence, we obtain the following result (cf. [13]).

COROLLARY 5.3. If $X_{\ell} \in \mathscr{G}(X)$, then $X_{\ell}^{\phi(t) / 2} \simeq X^{\phi(t) / 2}$, where $\phi$ denotes Euler's totient function. 
6. Appendix: on the structure of certain nilpotent groups. In this appendix, we sharpen [8, Theorem 1.1] which asserts that, in the notation of Section 2, with $r \geq 2$, we must have $t_{1}=1$ or 2 .

First, we point out that the condition that (2.1) splits, is not required in the conclusion of the theorem. This condition appeared (implicitly) in [8, proof of Lemma 2.1], in the description of the terms of the lower central series of $N$. However, we know that $N$ is nilpotent if and only if $T N$ is $F N$-nilpotent (since $T N, F N$ are commutative); and, even without the splitting hypothesis, the description given in fact describes the "lower central" $F N$-series of $T N$. Thus the conclusion of [8, Lemma 2.1] still holds.

On the other hand, it does not follow, without the splitting hypothesis, from the fact that $t_{1}=1$ or 2 that $\mathscr{G}(N)$ is trivial. For the calculation of $\mathscr{G}(N)$ as $\left(\mathbb{Z} / t_{1}\right)^{*} /\{ \pm 1\}$, carried out in [1], makes essential use of the splitting hypothesis. Thus it remains an interesting open problem to determine $\mathscr{G}(N)$ where $N$ is a finitely generated nilpotent group satisfying hypothesis (i) and (iii) of Section 1 . Further, the construction of a space $X$ satisfying $N \cong\left[S^{1}, \Omega X\right]_{\text {fr }}$ requires the splitting hypothesis.

In fact, we can substantially improve on the conclusion of [8, Theorem 1.1], even without the splitting hypothesis. We recall that we express $m=\exp T N$ as

$$
m=p_{1}^{n_{1}} p_{2}^{n_{2}} \cdots p_{\lambda}^{n_{\lambda}}
$$

where $p_{1}<p_{2}<\cdots<p_{\lambda}$ are primes. We then consider two cases. Case 2 (the exceptional case) is given by $p_{1}=2, n_{1} \geq 3$; while Case 1 (the general case) is simply the complement of Case 2. We now argue just as in [8], except that we consider the pairs $\left(t_{i}, t_{i+1}\right), i=1,2, \ldots, r-1$, instead of just the pair $\left(t_{1}, t_{2}\right)$. By doing so, and by incorporating the argument implicit in [8] that $r \nsupseteq 3$ if $t=2$, we conclude the following theorem.

THEOREM 6.1. (i) In the general case, with $r \geq 2$,

$$
\left(t_{1}, t_{2}, \ldots, t_{r}\right)=\left(1,1, \ldots, 1, t_{r}\right) .
$$

(ii) In the exceptional case, with $r \geq 2$,

$$
\left(t_{1}, t_{2}, \ldots, t_{r}\right)=\left(1,1, \ldots, \varepsilon, t_{r}\right), \quad \text { where } \varepsilon=1 \text { or } 2 .
$$

Of course, the conclusion of Theorem 6.1 is entirely consistent with the statement in [8] that $t$ can take any value if $r=1$.

\section{REFERENCES}

[1] C. Casacuberta and P. Hilton, Calculating the Mislin genus for a certain family of nilpotent groups, Comm. Algebra 19 (1991), no. 7, 2051-2069. MR 92j:20032. Zbl 742.20034.

[2] P. Hilton, On the genus of nilpotent groups and spaces, Israel J. Math. 54 (1986), no. 1, 1-13. MR 87m:55011. Zbl 602.20030.

[3] P. Hilton and G. Mislin, On the genus of a nilpotent group with finite commutator subgroup, Math. Z. 146 (1976), no. 3, 201-211. MR 53\#615. Zbl 311.20013.

[4] P. Hilton, G. Mislin, and J. Roitberg, Localization of Nilpotent Groups and Spaces, NorthHolland Mathematics Studies, no. 15. Notas de Matemática, no. 55 [Notes on Mathematics, no. 55], North-Holland Publishing Co., Amsterdam, Oxford; American Elsevier Publishing Co., Inc., New York, 1975. MR 57\#17635. Zbl 323.55016. 
[5] P. Hilton and D. Scevenels, Calculating the genus of a direct product of certain nilpotent groups, Publ. Mat. 39 (1995), no. 2, 241-261. MR 97b:20049. Zbl 849.20021.

[6]___ Calculating and interpreting the Mislin genus of a special class of nilpotent spaces, Proc. Amer. Math. Soc. 127 (1999), no. 11, 3433-3438. MR 2000b:55008. Zbl 938.55019.

[7] P. Hilton and C. Schuck, Calculating the genus of certain nilpotent groups, Bol. Soc. Mat. Mexicana (2) 37 (1992), no. 1-2, 263-269, Papers in honor of José Adem (Spanish). MR 95m:20037. Zbl 832.20055.

[8] - On the structure of nilpotent groups of a certain type, Topol. Methods Nonlinear Anal. 1 (1993), no. 2, 323-327. MR 94i:20061. Zbl 794.20046.

[9] _ Non-cancellation phenomena in a class of finitely generated nilpotent groups, Algebra (Krasnoyarsk, 1993) (Berlin) (Y. Ershov, ed.), de Gruyter, 1996, Proceedings of the Third International Conference held at Krasnoyarsk State University, Krasnoyarsk, August 23-28, 1993, pp. 93-101. MR 97g:20034. Zbl 851.20031.

[10] C. A. McGibbon, On the localization genus of a space, Algebraic Topology: New Trends in Localization and Periodicity. Barcelona Conference on Algebraic Topology (BCAT) (Sant Feliu de Guíxols, 1994) (Basel) (C. Broto, ed.), Progress in Mathematics, vol. 136, Birkhäuser, 1996, pp. 285-306. MR 97d:55019. Zbl 854.55008.

[11] G. Mislin, Nilpotent groups with finite commutator subgroups, Localization in Group Theory and Homotopy Theory, and Related Topics (Sympos., Battelle Seattle Res. Center, Seattle, Wash., 1974) (Berlin, Heidelberg, New York), Lecture Notes in Math., vol. 418, Springer, 1974, pp. 103-120. MR 50\#10080. Zbl 302.20029.

[12] G. Peschke, H-semidirect products, Canad. Math. Bull. 30 (1987), no. 4, 402-411. MR 89a:55009. Zbl 633.55007.

[13] A. Zabrodsky, $p$ equivalences and homotopy type, Localization in Group Theory and Homotopy Theory, and Related Topics (Sympos., Battelle Seattle Res. Center, Seattle, Wash., 1974) (Berlin, Heidelberg, New York), Lecture Notes in Math., vol. 418, Springer, 1974, pp. 161-171. MR 51\#14036. Zbl 302.55005.

Peter Hilton: Department of Mathematical Sciences, SUNY, Binghamton, NY 139026000, USA

Current address: Department of MATHematics, University of Central Florida, ORLANDO, FL 32816-1364, USA

E-mail address: marge@math . binghamton . edu

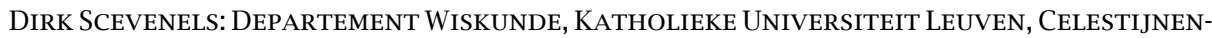
LAAN 200 B, B-3001 HEVERLEE, BELGIUM

E-mail address: di rk.scevene1s@wi s.ku1euven.ac.be 


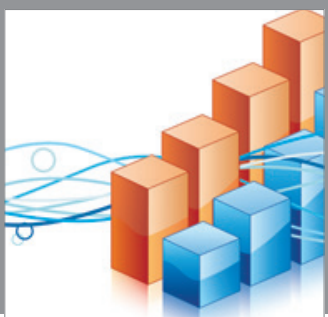

Advances in

Operations Research

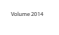

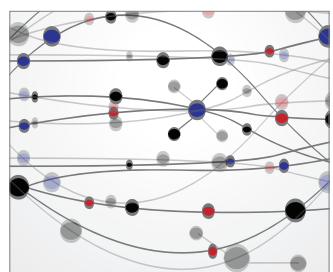

\section{The Scientific} World Journal
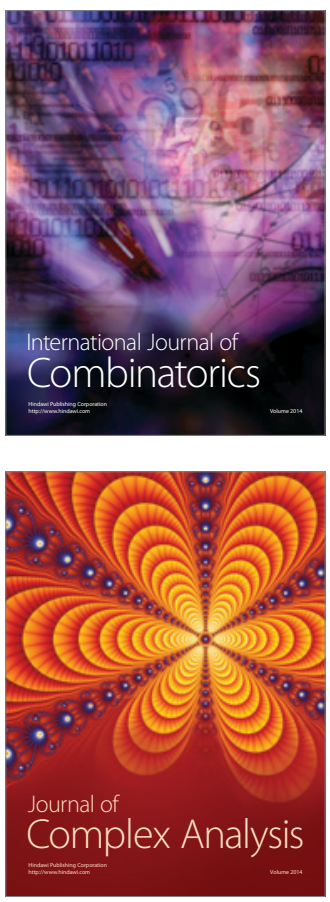

International Journal of

Mathematics and

Mathematical

Sciences
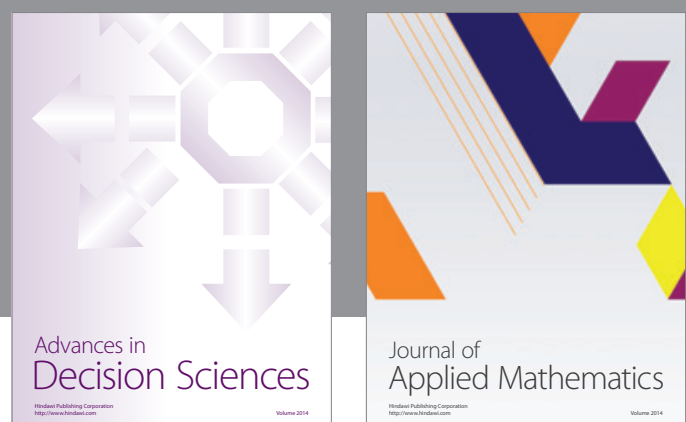

Journal of

Applied Mathematics
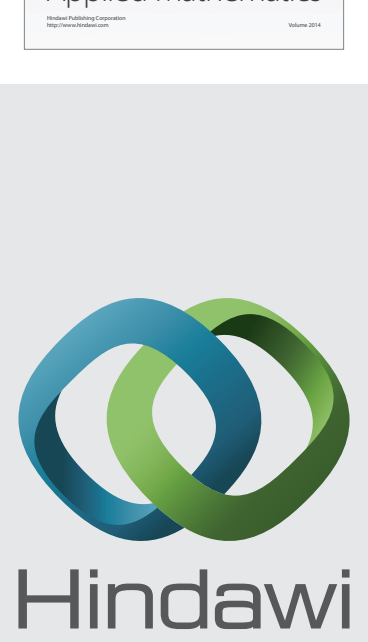

Submit your manuscripts at http://www.hindawi.com
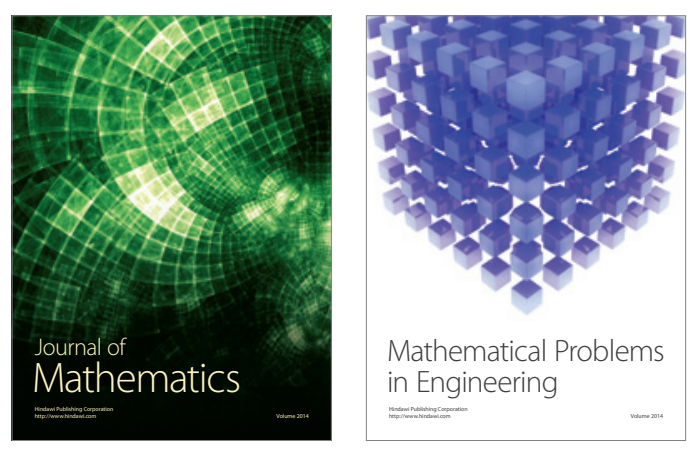

Mathematical Problems in Engineering
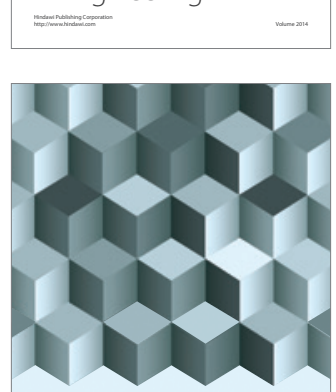

Journal of

Function Spaces
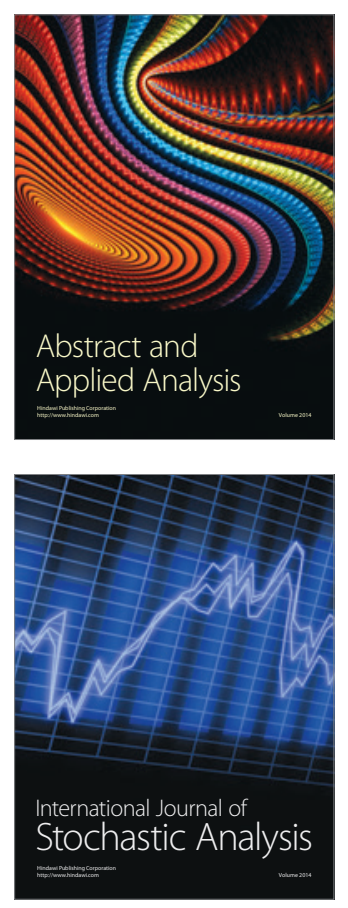

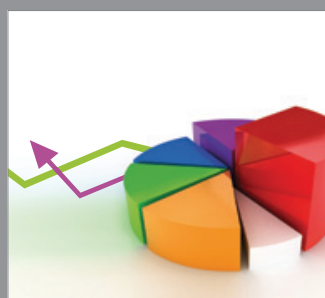

ournal of

Probability and Statistics

Promensencen
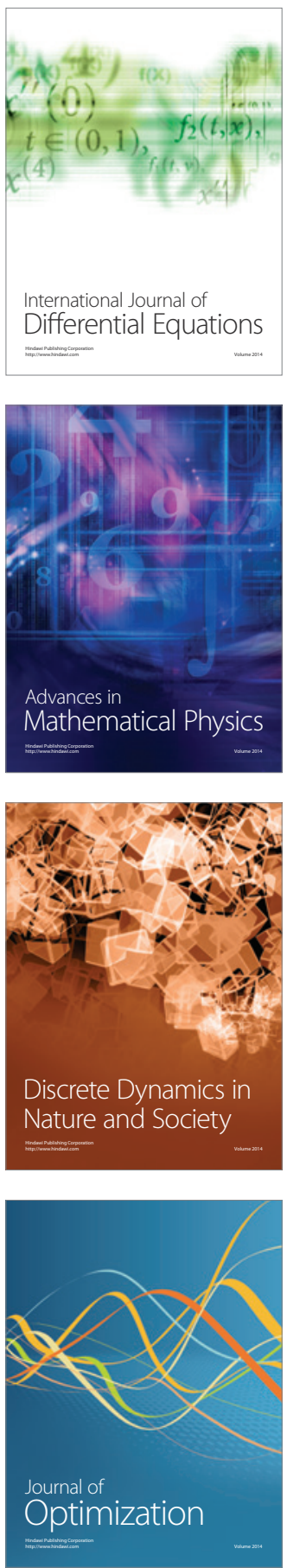